Ingénieurs et physiciens

s'intéressent depuis plusieurs

siècles au comportement

des liquides volatils sur des

plaques chaudes, et à la

lévitation du liquide sur

un coussin de vapeur, ou

caléfaction, qui en résulte.

Plusieurs phénomènes

nouveaux ont été récemment

décrits, en particulier si l'on

texture le solide (friction

spéciale, autopropulsion).

Mais ces systèmes sont aussi

un moyen de produire une

situation modèle, où l'on

manipule ou place sous

champ un liquide détaché

de son support.

\title{
La caléfaction
}

Baptiste Darbois-Texier, Guillaume Dupeux, Guillaume Lagubeau, Marie Le Merrer, Keyvan Piroird, Dan Soto, Christophe Clanet et David Quéré (quere@pmmh.espci.fr)

Physique et Mécanique des Milieux Hétérogènes, UMR 7636 du CNRS, ESPCI, 75231 Paris Cedex 05

et Ladhyx, UMR 7646 du CNRS, École polytechnique, 91128 Palaiseau Cedex

\section{Découverte de la caléfaction}

On a pu découvrir l'existence de la caléfaction dans l'enfance, dans les pages de Michel Strogoff (1876), quand Jules Verne aveugle son héros en exposant ses yeux à une épée chauffée à blanc. Le traitre responsable de cette torture prend alors le dessus, jusqu'au coup de théâtre final, quand nous découvrons que Strogoff a simulé sa cécité - ce qui lui permet de terrasser son ennemi, dans leur ultime combat. Comme l'explique Jules Verne, Strogoff a été sauvé par ses larmes, qui en se vaporisant à l'approche de la lame ont créé un film isolant et protecteur. À l'époque de l'écriture du roman, l'effet était connu : décrit une première fois en 1734 par Herman Boerhaave à Leiden, il avait fait ensuite l'objet du célèbre chapitre XV du Traité sur les propriétés de l'eau commune de Johann Gotlob Leidenfrost (1715-1794), publié en 1756 à Duisburg. Leidenfrost avait remarqué que l'eau, versée dans une cuillère polie et rougie au feu, "n'adhère pas à la cuillère " mais "y repose paisiblement, sans mouvement apparent et sans bouillir». Mieux encore, ces gouttes «ne s'évaporent que lentement, survivant une demi-minute au moins avant de disparaitre avec un léger craquement".

Leidenfrost avait bien compris la cause de cet état sphéroïdal, comme on l'a parfois appelé : comme pour l'épée de Jules Verne, il n'y a pas contact entre le solide brûlant et le liquide, qui s'évapore à son voisinage et lévite ainsi sur un coussin de vapeur. L'absence de contact rend ces gouttes extrêmement mobiles (ce sont des aéroglisseurs) et empêche l'ébullition : les bulles apparaissent habituellement sur les défauts du solide, et détacher un liquide de son support l'éloigne de ces sites de nucléation. En plaçant une source de lumière derrière les gouttes lévitantes,
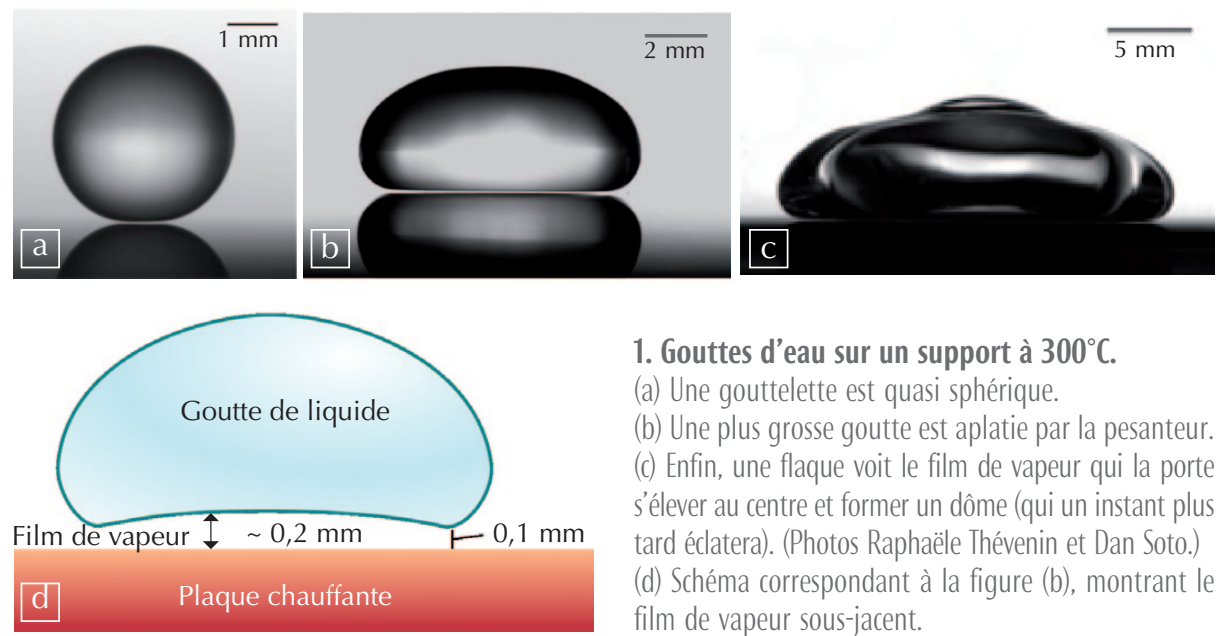

1. Gouttes d'eau sur un support à $300^{\circ} \mathrm{C}$. (a) Une gouttelette est quasi sphérique.

(b) Une plus grosse goutte est aplatie par la pesanteur. s'élever au centre et former un dôme (qui un instant plus tard éclatera). (Photos Raphaële Thévenin et Dan Soto.) (d) Schéma correspondant à la figure (b), montrant le film de vapeur sous-jacent. (c) Enfin, une flaque voit le film de vapeur qui la porte 


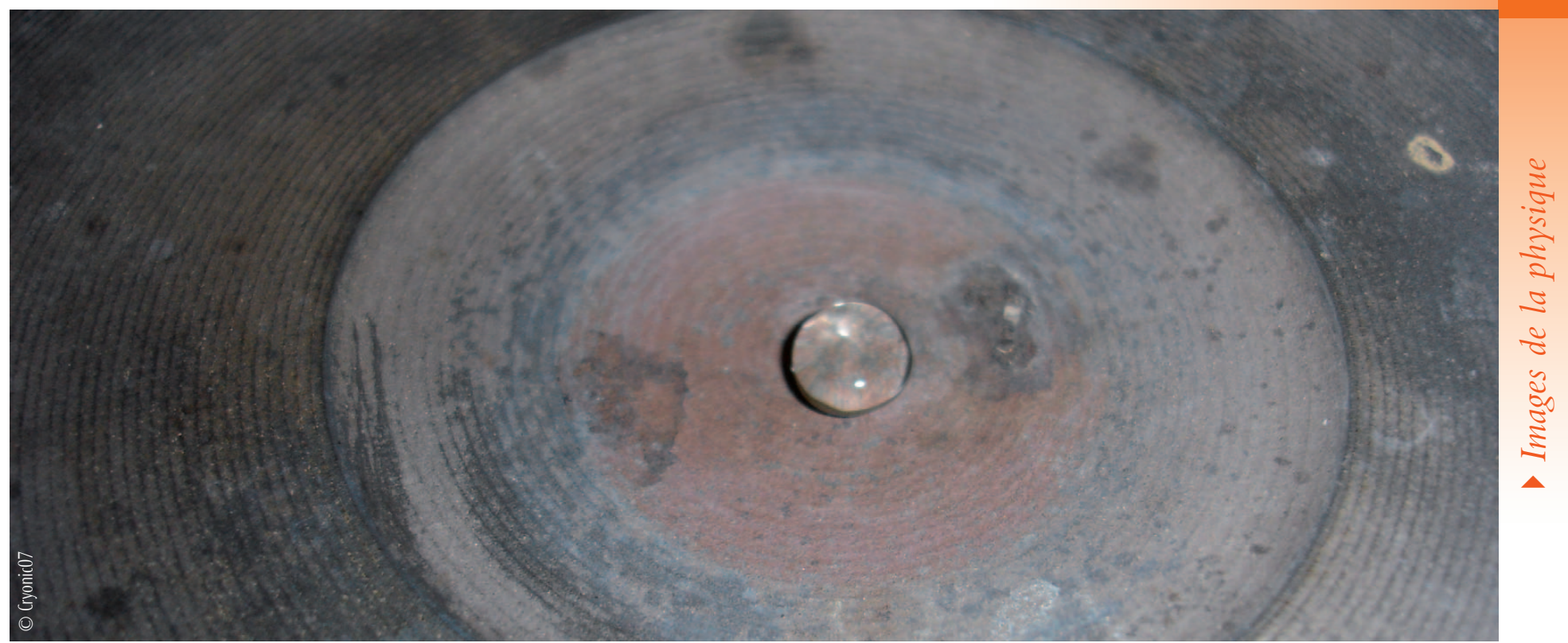

Effet Leidenfrost : goutte d'eau en caléfaction sur une plaque de cuisson.

Leidenfrost put évaluer à l'œil nu l'épaisseur du film, 100 micromètres environ. On a compris depuis que cette épaisseur résulte d'un compromis entre deux actions antagonistes : en s'évaporant, le liquide nourrit le film de vapeur et le fait s'épaissir ; dans le même temps, il appuie sur ce film, qui s'échappe par les côtés et s'amincit : l'égalité de ces flux contraires définit l'épaisseur du film sous-jacent.

On peut s'interroger sur la stabilité d'une telle situation : mille fois plus légère que le liquide qu'elle supporte, la vapeur tend à s'élever. Elle pourrait engendrer une colonne de bulles ascendantes, mais la tension de surface du liquide s'oppose en général aux déformations de l'interface que des bulles produiraient : quand une goutte est petite, le film de vapeur est stable. Toutefois, si c'est une grande flaque de Leidenfrost que l'on considère, on voit bien monter au centre de la flaque
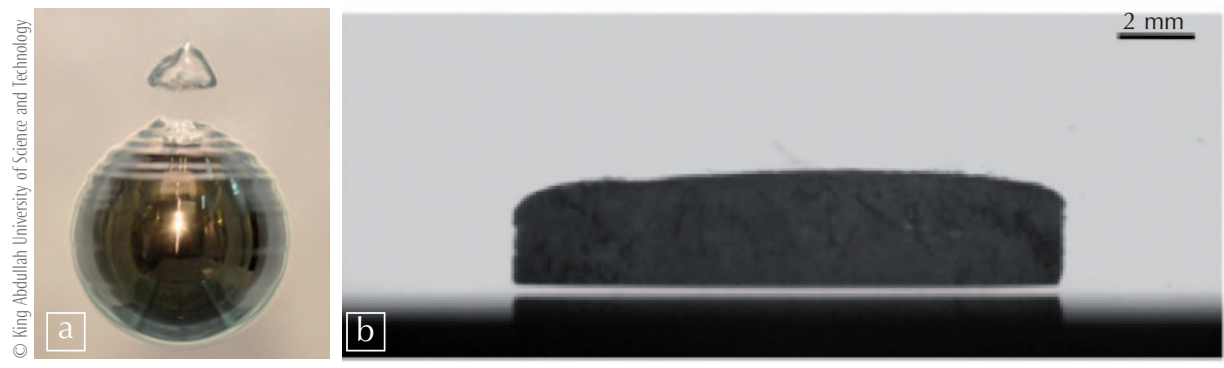

2. Autres situations de caléfaction. a) Bille d'acier (diamètre de $15 \mathrm{~mm}$ ) maintenue magnétiquement dans un bain de $\mathrm{FC}-72$, un liquide fluoré dont le point d'ébullition est de $56^{\circ} \mathrm{C}$. Quand la sphère est à une température supérieure à $130^{\circ} \mathrm{C}\left(200^{\circ} \mathrm{C}\right.$, ici), elle se couvre d'un film continu de vapeur qui remonte vers le pôle Nord de la bille, et y produit régulièrement des bulles. (Photo Ivan Vakarelski et Siggi Thoroddsen.) b) Un morceau de carboglace, qui se sublime à pression atmosphérique, peut également léviter, si son support est assez chaud (300 Cici). (Photo Raphaële Thévenin et Dan Soto.) une cheminée de vapeur qui crève son sommet : à grande échelle, la gravité (incarnée ici par Archimède) domine les forces de surface.

Ainsi, les formes de goutte et de film sous-jacent sont une affaire d'échelle : pour un rayon $r$ plus petit que la longueur capillaire $^{(1)}$ a pour laquelle forces de surface et poids sont comparables $(a=2,5 \mathrm{~mm}$ pour l'eau à son point d'ébullition), une goutte est quasi sphérique et repose sur une interface liquide/vapeur plane (fig. 1a) ; autour de la longueur capillaire $(r \sim a)$, la gravité écrase le liquide (fig. 1b), et le film sous-jacent se bombe légèrement (fig. 1d) ; à plus grande échelle, une flaque se troue sous l'effet de la montée de vapeur : le film est instable (fig. 1c).

Deux autres situations de caléfaction méritent d'être citées.

1) Si un solide très chaud est placé dans un bain de liquide volatil, un film de vapeur le couvre et l'isole du liquide environnant. Cet effet, parfois qualifié d'effet Leidenfrost inverse, a été décrit pour la première fois par Faraday en 1828. On le voit sur la figure $2 \mathrm{a}$ : une sphère centimétrique d'acier est portée à $200^{\circ} \mathrm{C}$ dans un liquide fluoré (dont le point d'ébullition est à $56^{\circ} \mathrm{C}$ ) et maintenue magnétiquement; un film de vapeur la recouvre et son écoulement (vers le pôle Nord de la sphère) produit des rides et le détachement périodique de bulles. Au bout d'un moment, la sphère se refroidit assez pour que l'ébullition se produise. Comme pour une goutte, mais pour des raisons bien différentes, une sphère ainsi couverte de vapeur sera ultra-mobile : elle tombe dans son bain à des vitesses deux à trois fois supérieures à ce que l'on observerait à $20^{\circ} \mathrm{C}$, en l'absence de film, comme l'a montré Thoroddsen en 2011.

2) Sur un solide chaud, on peut faire léviter des solides, plutôt que des liquides : il suffit pour cela de travailler avec des corps qui se subliment à pression atmosphérique, comme le fait la carboglace. Sa température de sublimation est de $-80^{\circ} \mathrm{C}$, si bien qu'un morceau de carboglace lévite sur un coussin de sa propre vapeur, si le support est assez chaud (figure 2b). Ces palets sont donc ultra-mobiles, et leur géométrie à la fois très simple (la surface sous-jacente reste plane quelle que soit l'échelle du palet) et façonnable (on peut les sculpter) rend ce système particulièrement attrayant pour conduire des études sur les phénomènes de caléfaction.

>> 


\section{Caléfaction sur des textures}

L'absence de friction des liquides caléfiés est la propriété la plus immédiatement frappante de ces systèmes. Qui n'a jamais vu des gouttes d'azote liquide roulant sur le sol jusqu'à atteindre le côté opposé de la pièce où on les a lancées ? On devine deux sources de résistance au mouvement rapide de ces gouttes. Il existe d'abord une force liée au frottement dans le coussin de vapeur, mais la faible viscosité de la vapeur et son épaisseur significative rendent en général négligeable cette friction visqueuse. En second lieu, le liquide déplace l'air environnant, comme le fait une goutte de pluie en chute libre, d'où il résulte une force de résistance inertielle. Cette force, proportionnelle à la densité de l'air, au carré de la vitesse et à la surface de la sphère, sera banalement de l'ordre du micronewton, cent fois plus faible que le poids de la goutte !

Une friction aussi ténue suggère deux axes de recherche : on doit se demander comment ralentir et même arrêter ces gouttes ultra-mobiles; mais on peut aussi chercher à exploiter cette mobilité en imaginant des systèmes où une faible force propulsive permet de piloter le liquide à une vitesse appréciable. Il s'avère que placer des textures à la surface du substrat permet de réaliser ces deux propriétés. La figure $3 \mathrm{a}$ montre ainsi une surface crénelée à une échelle (en profondeur) nettement inférieure à l'épaisseur de la goutte, ici d'un facteur 5 .
Freinage des gouttes

Comme on le voit sur la figure $3 \mathrm{~b}$, des gouttes lancées sur cette surface s'arrêtent au bout de quelques centimètres à peine, au lieu de quelques mètres sur une surface plane : les créneaux ont permis de multiplier par 100 le frottement du liquide!

Cette friction spéciale est bien différente de celle qui s'oppose au mouvement des gouttes sur un morceau de plastique ou une vitre. Comme on le voit sur la figure $3 a$, le liquide se déforme dans les creux de la texture, si bien que des bourrelets frappent (sans pour autant établir de contact) les côtés des créneaux. Or, ces chocs sont d'autant plus mous que la vitesse du liquide est grande. On a ainsi montré dans des expériences d'impact en caléfaction que si le solide chaud renvoie le liquide qui le frappe, le choc est d'autant moins élastique que l'énergie cinétique est grande par rapport à l'énergie de surface du système : le liquide se déforme à l'impact, ce qui lui permet de se comporter comme un ressort ; mais une partie de l'énergie élastique stockée en surface se transforme en énergie vibrationnelle, perdue pour le mouvement d'ensemble du liquide. Pour les créneaux, il doit en être de même, et l'on comprend ainsi que la perte d'énergie (et donc la friction) soit pilotée par la masse volumique $\rho$ du liquide, et non par celle de l'air - expliquant le fort ralentissement sur les créneaux observé sur la figure $3 b$.

Un bref raisonnement en loi d'échelle permet de préciser comment la forme des créneaux pilote le freinage des gouttes. La masse d'un bourrelet s'écrit $\rho H \lambda R$, en suivant les notations de la figure $3 a$; supposer un choc mou sur le côté du créneau revient à supposer une décélération d'ordre $V^{2} / \lambda$ de cette masse, et donc une friction totale (intégrée sur les $R / \lambda$ bourrelets présents sous la goutte) d'ordre $\rho V^{2} H R^{2} / \lambda$, qui a bien la propriété de s'annuler quand les créneaux s'évanouissent $(H \rightarrow 0$ ou $\lambda \rightarrow \infty)$. Si l'on équilibre cette force par l'inertie du liquide, d'ordre $\rho R^{3} \mathrm{~d} V^{2} / \mathrm{d} x$, on attend bien le comportement exponentiel observé sur la figure $3 b$, avec une longueur caractéristique de décélération $H R / \lambda$, fonction du dessin des créneaux et de la taille de la goutte.

\section{Autopropulsion des gouttes}

Cependant, des textures peuvent induire des propriétés plus remarquables encore. En 2006, Heiner Linke a ainsi montré qu'un liquide posé en caléfaction sur un support couvert de dents asymétriques est autopropulsé dans la direction de la pente descendante des dents (fig. 4). Au bout de quelques centimètres de course, il atteint une vitesse terminale de l'ordre de $10 \mathrm{~cm} / \mathrm{s}$, réalisant ainsi un système original de refroidisseur où du liquide emmène (un peu de) la chaleur de son support sans l'emploi d'une pompe ou de tout autre dispositif mécanique.

Les mesures faites (en attrapant les gouttes avec un fil et en observant, tel le pêcheur à la ligne, la déflection du fil due à la force
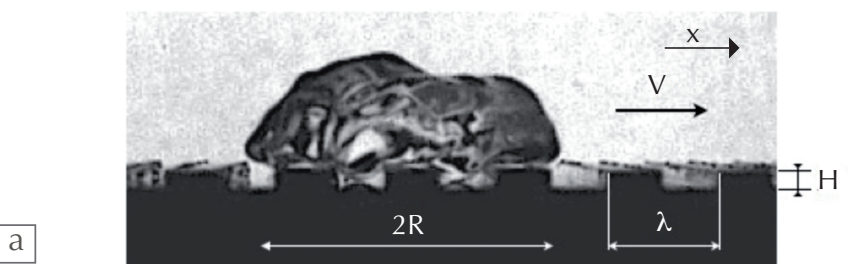

$\mathrm{b}$

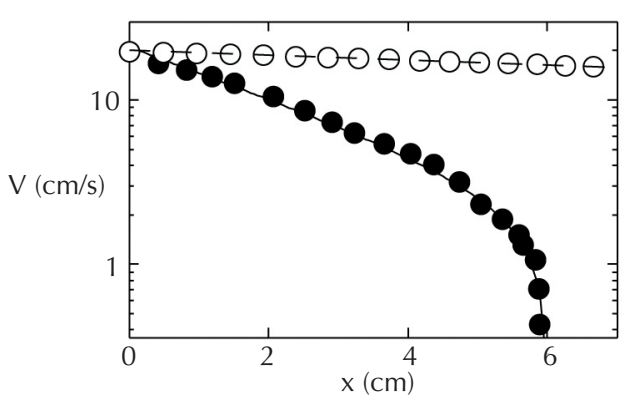

3. Goutte caléfiée d'éthanol avançant sur une surface crénelée chauffée à $450^{\circ} \mathrm{C}$ $(H=480 \mu \mathrm{m}-\lambda=3 \mathrm{~mm}-2 R=8 \mathrm{~mm})$. À cause des déformations de la goutte, sa vitesse $V$ décroît exponentiellement avec la distance parcourue x (points noirs), jusqu'à s'arrêter brutalement (chute de la vitesse autour de $x=6 \mathrm{~cm}$ ). Une goutte sur une surface plane (points blancs) parcourt, quant à elle, plusieurs mètres avant de ralentir de manière significative.

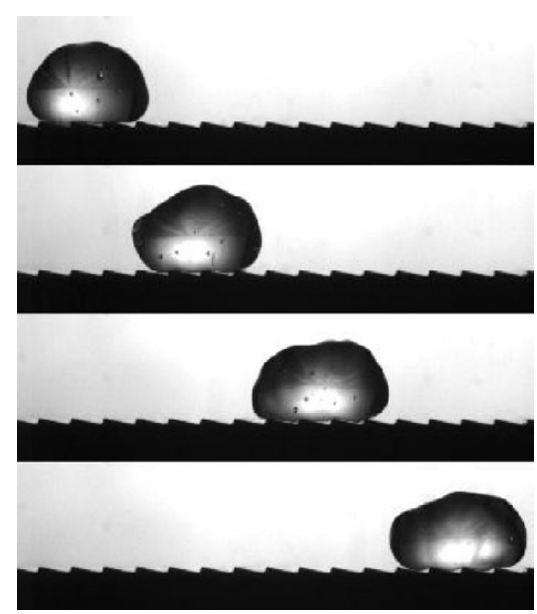

4. Expérience de linke : une goutte (de rayon $R=2 \mathrm{~mm}$ ) est placée sur un "ratchet" (solide à la surface en toit d'usine), dont les dents ont une profondeur $H=0,2 \mathrm{~mm}$ et une longueur $\lambda=1,5 \mathrm{~mm}$. Si le solide est assez chaud pour induire de la caléfaction, le liquide est autopropulsé dans la direction où il rencontre la partie abrupte des dents. L'intervalle entre les photos est de $40 \mathrm{~ms}$. (Photos Marie Le Merrer.) 

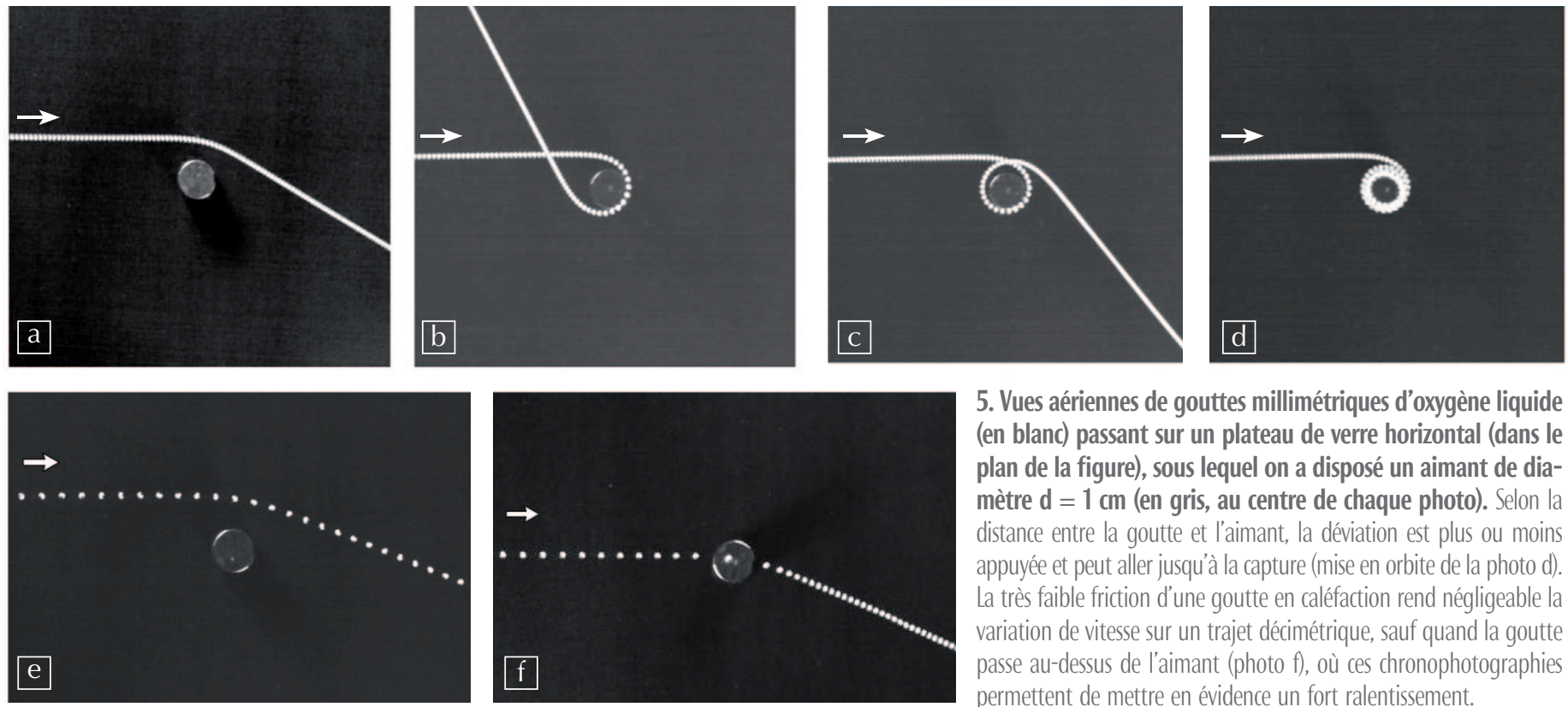

5. Vues aériennes de gouttes millimétriques d'oxygène liquide (en blanc) passant sur un plateau de verre horizontal (dans le plan de la figure), sous lequel on a disposé un aimant de diamètre $\mathrm{d}=1 \mathrm{~cm}$ (en gris, au centre de chaque photo). Selon la distance entre la goutte et l'aimant, la déviation est plus ou moins appuyée et peut aller jusqu'à la capture (mise en orbite de la photo d). La très faible friction d'une goutte en caléfaction rend négligeable la variation de vitesse sur un trajet décimétrique, sauf quand la goutte passe au-dessus de l'aimant (photo f), où ces chronophotographies permettent de mettre en évidence un fort ralentissement.

exercée par la prise) indiquent des forces de l'ordre de $10 \mu \mathrm{N}$, soit quelques pourcents seulement du poids du liquide. On voit des ondes se propager le long du liquide, on observe des petites oscillations de la goutte, on remarque des bourrelets sous le liquide, sans parler des légères différences de température (et donc de tension de surface) entre le haut et le bas de la goutte - chacun de ces faits pouvant potentiellement conduire à un scénario de propulsion. De ce point de vue, une avancée significative fut de réaliser qu'un solide de Leidenfrost (tel que le montre la figure 2b) avance aussi sur une surface chaude avec des rugosités en forme de toit d'usine, et dans la même direction qu'une goutte : la propulsion a lieu sans que l'objet soit déformable.

On est ainsi amené à comprendre que le point essentiel est la production de vapeur sous la goutte, une vapeur évacuée par la pression qu'exerce le liquide sur le film qui le supporte. Si cet écoulement est isotrope sur un solide plan (cas de la figure 1), il peut ne plus l'être sur le solide aux textures asymétriques de la figure 4. En disposant des microbilles de silice sur les textures, et en approchant un disque de carboglace, on confirme qu'il y a bien rectification de l'écoulement de vapeur : cette dernière est évacuée dans la direction des pentes descendantes des dents (celle-là même choisie par le liquide), puis tourne à $90^{\circ}$ en heurtant la marche, ce qui lui permet de s'évacuer le long de ces gouttières naturelles, perpendiculairement à la direction du liquide. Ce dernier est donc tiré par la force visqueuse qu'induit l'écoulement descendant de la vapeur ; cette force est certes faible (la viscosité de la vapeur est trente fois inférieure à celle de l'eau et la vitesse de la vapeur, révélée par le mouvement des marqueurs, est de l'ordre de $10 \mathrm{~cm} / \mathrm{s}$ ), mais suffisante sur ces systèmes lévitant pour entrainer à des vitesses significatives le liquide (ou le solide) au-dessus. On comprend ainsi que la locomotion est activée dès que l'on canalise la vapeur dans une direction donnée - ce que fait la vapeur sur les dents à cause de leur asymétrie, mais que n'importe quel dispositif forçant un écoulement anisotrope doit réaliser. On peut aussi imaginer des dessins de textures permettant la rotation du liquide plutôt que sa translation, et c'est dans ces différentes voies que nous travaillons actuellement.

\section{Caléfaction sous champ}

Comme on l'a vu, la présence de textures sur un solide permet le contrôle des gouttes caléfiées, puisque l'on peut induire aussi bien des ralentissements et des arrêts que des mouvements directionnels. Il est intéressant de se demander si, au-delà de cette première famille de possibilités, d'autres formes de contrôle peuvent être induites par l'application d'un champ, électrique ou magnétique, par exemple. Nous développons ici ce second cas, en discutant ce qui se passe avec l'oxygène liquide, en caléfaction à $25^{\circ} \mathrm{C}$ et paramagnétique.

Une première expérience est simple : on lance à une vitesse contrôlée de l'oxygène liquide sur un plateau de verre sous lequel on a disposé un aimant. En jouant sur la force de l'aimant et sur sa distance au verre, on module la profondeur du piège magnétique ainsi constitué, et une goutte d'oxygène placée là y est piégée. Mais l'oxygène en mouvement a de l'inertie, et il pourra, si son énergie cinétique se compare à l'énergie magnétique du piège, voir sa trajectoire modifiée par l'aimant, comme en témoigne la figure 5.

Pour reprendre le titre de Queneau, on dispose ainsi d'une véritable petite cosmogonie portative : la trajectoire du liquide est plus ou moins perturbée selon sa distance à l'aimant, avec toutes sortes d'angles de déviation - aux vitesses de la figure 5, cet angle peut même quasi diverger pour une " périhélie » de l'ordre de la taille de l'aimant : on peut ainsi piéger le liquide, qui se met à orbiter autour de son attracteur (fig. 5d). Cette façon qu'a le liquide d'interagir avec le puits de potentiel évoque ce qui se passe quand une balle de basket ou de golf tourne autour du pot (pour ainsi dire) avant d'y tomber, ou au contraire de s'en extraire. On peut enfin accélérer le liquide en le faisant passer près d'un aimant en mouvement, une idée inspirée de l'effet de fronde gravitationnelle utilisée par la sonde Voyager pour gagner de l'impulsion au voisinage des planètes qu'elle croise.

Ces systèmes sont aussi utiles pour modéliser ce qui se passe lorsque le liquide passe au-dessus de l'aimant plutôt qu'au large de ce dernier. Si l'on observe aussi une déviation (sauf quand l'oxygène passe strictement au centre du piège), elle 
$>>>$

s'accompagne d'une nette décélération du liquide (fig. 5f). Ce dernier, en tombant dans le puits d'énergie, se déforme, s'aplatit et relaxe ensuite, ayant converti une part de son énergie en vibrations qui peu à peu s'atténuent. Cette question est très générale : comme on l'a dit plus haut, l'impact d'un liquide non-mouillant (comme il l'est en caléfaction) sur son support est d'autant plus mou qu'il est violent : plus le liquide s'écrase, et plus son énergie est transférée en vibrations, aux dépens de l'élasticité du choc. Une goutte caléfiée sous champ semble donc un système modèle pour comprendre cette question, et plus généralement pour décrire le comportement d'un liquide séparé de son support en présence d'une action externe. Par exemple, en faisant tourner l'aimant sur lui-même, on va mettre en rotation le liquide et pouvoir étudier les modes de déformation qui en résultent, un problème étudié théoriquement par Rayleigh, Poincaré et Chandrasekhar, mais pour lequel il existe aujourd'hui très peu de données.

\section{Futur de la caléfaction}

Deux cent cinquante ans après sa découverte, la caléfaction pose encore des problèmes nouveaux, comme nous avons tenté de le montrer dans cet article. La recherche sur ces sujets devrait se développer dans trois directions principales.

(1) La simple description du phénomène demande à être approfondie, notamment la forme de la poche de vapeur sous-jacente et son instabilité quand elle devient grande. Il convient en particulier de comprendre comment l'écoulement de vapeur modifie les seuils d'instabilités, comme l'a suggéré Snoeijer en 2009. Les mouvements internes dans une goutte caléfiée ont été très peu décrits, et leur relation avec la différence de température existant entre les deux pôles de la goutte reste à établir. La façon dont se construit le film au moment du contact, comme les derniers instants d'une goutte caléfiée, sont deux autres sujets qui méritent d'être étudiés.

(2) La caléfaction des liquides complexes est un autre sujet d'avenir. On cherche souvent à refroidir des plaques chaudes (dans un laminoir, par exemple) avec de l'eau, et la caléfaction, qui fait rebondir les gouttes sur le métal, est préjudiciable. Des liquides complexes pourraient avoir des comportements différents, que l'on pense à des solutions de polymères, de tensioactifs, ou à des émulsions.

(3) Induire une lévitation par la température est restrictif : un liquide non volatil ne lévitera pas, et on ne pourra pas toujours chauffer le support. Il est donc intéressant de se demander si l'on peut caléfier «à froid " un liquide. Une voie est la dynamique : en faisant osciller un bain d'huile, Yves Couder a montré qu'on peut faire léviter des gouttes d'huile à la surface de ce bain. Il est envisageable d'étendre ce principe à d'autres systèmes : ainsi, un solide se déplaçant rapidement entraîne avec lui un film d'air sur lequel un liquide (quelconque) pourra "surfer". Ces situations de "caléfaction" dynamique sont à nos yeux un domaine particulièrement prometteur. Et l'on comprend la richesse de ces systèmes où la conjonction du non-mouillage, des écoulements d'air ou de vapeur, et des propriétés interfaciales engendre quantité de situations nouvelles, rénovant de façon stimulante les observations du Docteur Leidenfrost.

(1) La longueur capillaire $a$ est définie par $a=(\gamma / \rho g)^{1 / 2}$, où $\gamma$ est la tension de surface de l'interface liquidevapeur, $\rho$ la masse volumique du liquide et $g$ l'accélération de la pesanteur.

\section{Bibliographie}

- J.G. Leidenfrost, "0n the fixation of water in diverse fire", Int. J. Heat Mass Trans., 9 (1966) 1153-1166. (Traduction de l'article original de 1756 par Carolyn Wares, Univ. of Oklahoma.)

- B.S. Gottfried et al., "The Leidenfrost phenomenon: film boiling of liquid droplets on a flat plate", Int. J. Heat Mass Trans., 9 (1966) 1167-1172.

- Y. Couder et al., "Les gouttes marcheuses ", Reflets de la physique, 5 (2007) 20-24.

- H. Linke et al., "Self-propelled Leidenfrost droplets", Phys. Rev. Lett., 96 (2006) 154502.

- J. H. Snoeijer et al., "Maximum size of drops levitated by an air cushion”, Phys. Rev. E, 79 (2009) 036307.

- G. Lagubeau et al., "Leidenfrost on a ratchet", Nature Physics, 7 (2011) 395.

- G. Dupeux et al., "Trapping Leidenfrost drops with crenelations", Phys. Rev. Lett., 107 (2011) 114503.

- M. Le Merrer et al., "Wave drag on floating bodies", PNAS, 108 (2011) 15064.

- G. Dupeux et al., "Viscous mechanism for Leidenfrost propulsion on a ratchet", EPL, 96 (2011) 58001.

- I.U. Vakarelski et al., “Drag Reduction by Leidenfrost Vapor Layers", Phys. Rev. Lett., 106 (2011) 214501.

- K. Piroird et al., "Magnetic control of Leidenfrost drops", Phys. Rev. E, 85 (2012) 056311. 